Communications in Physics, Vol. 22, No. 3 (2012), pp. 229-238

\title{
DIELECTRIC AND RELAXOR FERROELECTRIC PROPERTIES IN PZT-PMNN-PSBN CERAMICS
}

\author{
THAN TRONG HUY \\ Phu Xuan Hue University \\ NGUYEN DINH TUNG LUAN \\ Hue Industrial College \\ TRUONG VAN CHUONG \\ Hue Science University \\ LE VAN HONG \\ Institute of Material Science, VAST
}

\begin{abstract}
The rhombohedral perovskite composite $0.9 \mathrm{~Pb}\left(\mathrm{Zr}_{0.5} \mathrm{Ti}_{0.5}\right) \mathrm{O}_{3}-x \mathrm{~Pb}\left(\mathrm{Mn}_{1 / 3} \mathrm{Nb}_{2 / 3}\right) \mathrm{O}_{3}-$ (0.1-x) $\mathrm{Pb}\left(\mathrm{Sb}_{1 / 2} \mathrm{Nb}_{1 / 2}\right) \mathrm{O}_{3}, x=0.05 ; 0.06 ; 0.07$; 0.08; 0.09; 0.1 (PZT - PMnN - PSbN) was synthesized by the Columbite precursor route. The temperature dependence of dielectric constant and its loss in a frequency region of $0.1 \mathrm{kHz}-500 \mathrm{kHz}$ was measured in aim to search the weakfield dielectric response in PZT-PMnN-PSbN systems. A highest value of $\varepsilon_{\max } \dot{2} 20000$ was found at $1 \mathrm{kHz}$ with the temperature $T_{m}$ of around $575 \mathrm{~K}$. Using an extended Curie-Weiss law the diffuse phase transition was determined. Fitting by using Vogel - Fulcher and power relationship indicated that the polarization fluctuation above the static freezing temperature behaved like as a spin-glass one. Cole-Cole analyses showed the non - Debye type relaxation in the system studied.
\end{abstract}

\section{INTRODUCTION}

Perovskite based relaxor ferroelectric materials deserved a considerable interest due to their rich physical properties and various possible applications in memory storage devices, micro-electro-mechanical systems, multilayer ceramic capacitors and recently, in the area of opto-electronic devices [1-4]. In contrast to the normal ferroelectrics, they exhibit a strong frequency dispersion of the dielectric constant without the change in crystalline phase structure in the temperature region near $T_{m}$ (the temperature, at which the diffuse permittivity is maximum). Basically in the compositionally homogenous systems, the quenched random disorder causes a breaking of the long-range polar order in the unit cell level, leading to the broadening of the $\varepsilon^{\prime}(T)$ [5]. Such materials exhibit a slow enough relaxation dynamics and hence have been termed the ferroelectric relaxor [6-7]. Burns and Decol [8] have observed an existence of polar-regions in the relaxor at temperatures higher than $T_{m}$. In principle, the relaxors are classified in two families: The first is the lead manganese niobate (PMN) 1:2 family such as $\mathrm{Pb}\left(\mathrm{Mg}_{1 / 3} \mathrm{Nb}_{2 / 3}\right) \mathrm{O}_{3}$, and the second is the lead scandium niobate (PSN) 1:1 family such as $\mathrm{Pb}\left(\mathrm{Sc}_{1 / 2} \mathrm{Nb}_{1 / 2}\right) \mathrm{O}_{3}$. Similarly, the $\mathrm{PZT}-\mathrm{Pb}\left(\mathrm{Mg}_{1 / 3} \mathrm{Nb}_{2 / 3}\right) \mathrm{O}_{3}, \mathrm{PZT}-\mathrm{Pb}\left(\mathrm{Zn}_{1 / 3} \mathrm{Nb}_{2 / 3}\right) \mathrm{O}_{3}$ systems belong to the first family, and the $\mathrm{PT}-\mathrm{Pb}\left(\mathrm{Sc}_{1 / 2} \mathrm{Nb}_{1 / 2}\right)_{3}$ belongs to the second one. The like spin-glass state obeying 
Vogel-Fulcher law and the dielectric transition complied with the extended Curie-Weiss law were observed in these systems. The repord results indicated that the dielectric relaxation in these systems exhibits as a non-Debye type $[9,20,21]$. The relaxor behaviors of the ternary and quarternary compounds were also investigated and reported recently $[5,8,22,23]$.

In this paper, the relaxor behavior of $0.9 \mathrm{~Pb}\left(\mathrm{Zr}_{0.5} \mathrm{Ti}_{0.5}\right) \mathrm{O}_{3}-\mathrm{xPb}\left(\mathrm{Mn}_{1 / 3} \mathrm{Nb}_{1 / 3}\right) \mathrm{O}_{3}$ - (0.1-x) $\mathrm{Pb}\left(\mathrm{Sb}_{1 / 2} \mathrm{Nb}_{1 / 2}\right) \mathrm{O}_{3}$, (abbreviated by PZT-PMnN-PSbN or PZT-PMnSbN) with $\mathrm{x}=0.05 ; 0.06 ; 0.07 ; 0.08 ; 0.09 ; 0.1$ ceramics was investigated. The real and imaginary parts of the dielectric permittivity and loss in a frequency range of $0.1 \mathrm{kHz}-500 \mathrm{kHz}$ at a temperature range of $540 \mathrm{~K}-600 \mathrm{~K}$ has been analyzed. We have investigated the diffuse phase transition of the system by using the extended Curie - Weiss law and discovered that the dependence of $T_{m}$ values on frequency obey the power and Vogel-Fulcher relations but not with the Debye one. Fitting the experimental data by using the above relations and analyzing the Cole-Cole diagrams, we estimated the order-disorder and relaxation characteristics in this system.

\section{EXPERIMENTAL}

The polycrystalline samples of PZT-PMnN-PSbN were prepared by Coulombite precursor route using highly purified oxides, $\mathrm{PbO}$ (99.9\%), $\mathrm{ZrO}_{2}(99.9 \%), \mathrm{TiO}_{2}$ (99.9\%), $\mathrm{MnCO}_{3}(99.9 \%), \mathrm{Sb}_{2} \mathrm{O}_{3}(99.9 \%)$ and $\mathrm{Nb}_{2} \mathrm{O}_{5}(99.9 \%)$ being taken in stoichiometric ratios. First, the two finely mixed powders of $\left(\mathrm{MnCO}_{3}, \mathrm{Nb}_{2} \mathrm{O}_{5}\right)$ and $\left(\mathrm{Sb}_{2} \mathrm{O}_{3}, \mathrm{Nb}_{2} \mathrm{O}_{5}\right)$ were grinded by using a teflon-motar for $10 \mathrm{~h}$ in alcohol and then separately calcined at $1200^{\circ} \mathrm{C}$ in an alumina crucible for $3 \mathrm{~h}$ to create $\mathrm{MnNb}_{2} \mathrm{O}_{6}$ and $\mathrm{Sb}_{2} \mathrm{Nb}_{2} \mathrm{O}_{8}$, respectively. The calcined powders were then grinded and mixed by mortar again with $\mathrm{PbO}, \mathrm{ZrO}_{2}$ and $\mathrm{TiO}_{2}$ for $10 \mathrm{~h}$. An extra wt-7\% PbO was taken to compensate the lead lost during calcining and sintering. The finely mixed powder was calcined at $850^{\circ} \mathrm{C}$ for $2 \mathrm{~h}$, regrinded again for 20 $\mathrm{h}$ and pressed into the pellets with diameter of $12 \mathrm{~mm}$ and thickness of $1.4 \mathrm{~mm}$ by using poly-vinyl-alcohol (PVA) as the binder. The pellets were sintered at $1150^{\circ} \mathrm{C}$ for $2 \mathrm{~h}$ and then gradually cooled down to room temperature as following: It first decreased down from $1150^{\circ} \mathrm{C}$ to $800^{\circ} \mathrm{C}$ for $2 \mathrm{~h}$, then continuously from $800^{\circ} \mathrm{C}$ to $500^{\circ} \mathrm{C}$ for $1 \mathrm{~h}$ and finally from $500^{\circ} \mathrm{C}$ to room temperature. Surfaces of the sintered pellet were finely polished, cleaned in ultrasonic cleaner, then silver electrodeposited and numbered from M05 to M10, corresponding to $\mathrm{x}$ from 0,05 to 0,10 , respectively. The frequency dependence of dielectric constant and loss tangent were measured by using the Hioki LCR Hi Tester $3532-50$ in a frequency range of $0.1 \mathrm{kHz}-500 \mathrm{kHz}$ at a temperature range of $50^{\circ} \mathrm{C}$ to $350^{\circ} \mathrm{C}(320 \mathrm{~K}$ to $625 \mathrm{~K})$. The temperature dependence of dielectric constant was recorded with a heating rate of $0.5 \mathrm{~K} \cdot \mathrm{min}^{-1}$.

\section{RESULTS AND DISCUSSION}

\section{III.1. Dielectric behavior of PZT-PMnN-PSbN system}

Fig. 1 showed the temperature dependence of the real part of electric permittivity $\left(\varepsilon^{\prime}\right)$ and the dissipation factor $(\tan \delta)$ at various frequencies. The plots show a strong frequency dispersion which exhibits the relaxor behavior of PZT-PMnN-PSbN ceramics. 
It was observed that the temperature $T_{m}$ of maximum permittivity of all samples shifted to higher temperatures while $\varepsilon_{\max }$ decreased and $(\tan \delta)_{\max }$ increased upon increasing frequency. Fig. 1 also showed that all samples have a diffuse phase transition in the transition temperature region.

The real $\left(\varepsilon^{\prime}\right)$ and imaginary $\left(\varepsilon^{\prime \prime}\right)$ parts of dielectric constant and loss tangent $(\tan \delta)$ can be calculated from the measured capacitance and phase values of the samples versus temperature. The maximum dielectric permittivity $\left(\varepsilon^{\prime} \max \right)$ at $1 \mathrm{kHz}$, its temperature $\left(T_{m}\right)$, and the fitting parameters using the modified Curie-Weiss law are listed in Table 1 . The value of $T_{m}$ increases with increasing of $\mathrm{PMnN}$ component, but the $\varepsilon^{\prime}$ max abnormally depends on $\mathrm{PMnN}$ component and has the maximum value as $\mathrm{x}=0.07$.

In order to examine the diffuse phase transition and relaxor properties, the following modified Curie-Weiss formula has been used for analyzing of experimental data:

$$
\frac{1}{\varepsilon}-\frac{1}{\varepsilon_{\max }}=\frac{\left(T-T_{m}\right)^{\gamma}}{C^{\prime}}
$$

or

$$
\log \left(\frac{1}{\varepsilon}-\frac{1}{\varepsilon_{\max }}\right)=\gamma \log \left(T-T_{m}\right)-\log C^{\prime}
$$

where $\mathrm{C}$ / is the modified Curie-Weiss constant, and $\gamma$ is the diffuseness exponent, which changes from 1 to 2 for normal ferroelectrics to fully disorder relaxor ferroelectrics, respectively. Equation (1) can be solved graphically using a log-log plot, as shown in Fig. 2.

The given value of $\gamma$ at $1 \mathrm{kHz}$ as presented in Table 1 is an evidence to suggest the diffuse phase transition (DPT) happened in the samples. It is expected that the disorder in the cation distribution (compositional fluctuations) causes the DPT where the local Curie points of different micro-regions are statistically distributed in a wide temperature range around the mean Curie point. The non-equality of phase transition temperature obtained from $\varepsilon(\mathrm{T})$ and $\tan \delta(\mathrm{T})$ measurement also confirms the existence of the DPT. It has shown that the value of the diffuseness, $\gamma$, increases with increasing of $\mathrm{PMnN}$ component. This indicates that, the disorder in $\mathrm{B}$ site in materials increases with increasing of $\mathrm{PMnN}$ component in the systems.

Table 1. The dielectric permittivity maximum $\left(\varepsilon^{\prime} \max \right)$ and its temperature $\left(T_{m}\right)$, and the fitting parameters to the modified Curie-Weiss law.

\begin{tabular}{lccccccc}
\hline Sample & $\varepsilon$ & $\tan \delta$ & $\varepsilon^{\prime} \max$ & $\begin{array}{l}\mathbf{T}_{m} \\
(\mathbf{K})\end{array}$ & $\gamma$ & $\begin{array}{l}\mathbf{C}^{\prime} \times \mathbf{1 0}^{5} \\
(\mathbf{K})\end{array}$ & $\begin{array}{l}\mathbf{T}_{B} \\
(\mathbf{K})\end{array}$ \\
\hline M05 & 1520 & 0.03 & 24085 & 555 & 1.4481 & 5.123 & 596 \\
M06 & 1537 & 0.01 & 24488 & 557 & 1.5237 & 4.433 & 609 \\
M07 & 1555 & 0.008 & 29090 & 568 & 1.7689 & 6.783 & 615 \\
M08 & 1202 & 0.008 & 22689 & 572 & 1.8822 & 6.893 & 617 \\
M09 & 1101 & 0.012 & 17848 & 575 & 1.9241 & 5.773 & 620 \\
M10 & 980 & 0.009 & 15541 & 580 & 1.9678 & 3.983 & 625 \\
\hline
\end{tabular}

A common characteristic of all relaxors is the existence of disorder in crystalline structure. In principle the disorder is caused by variation in local electric field as well 

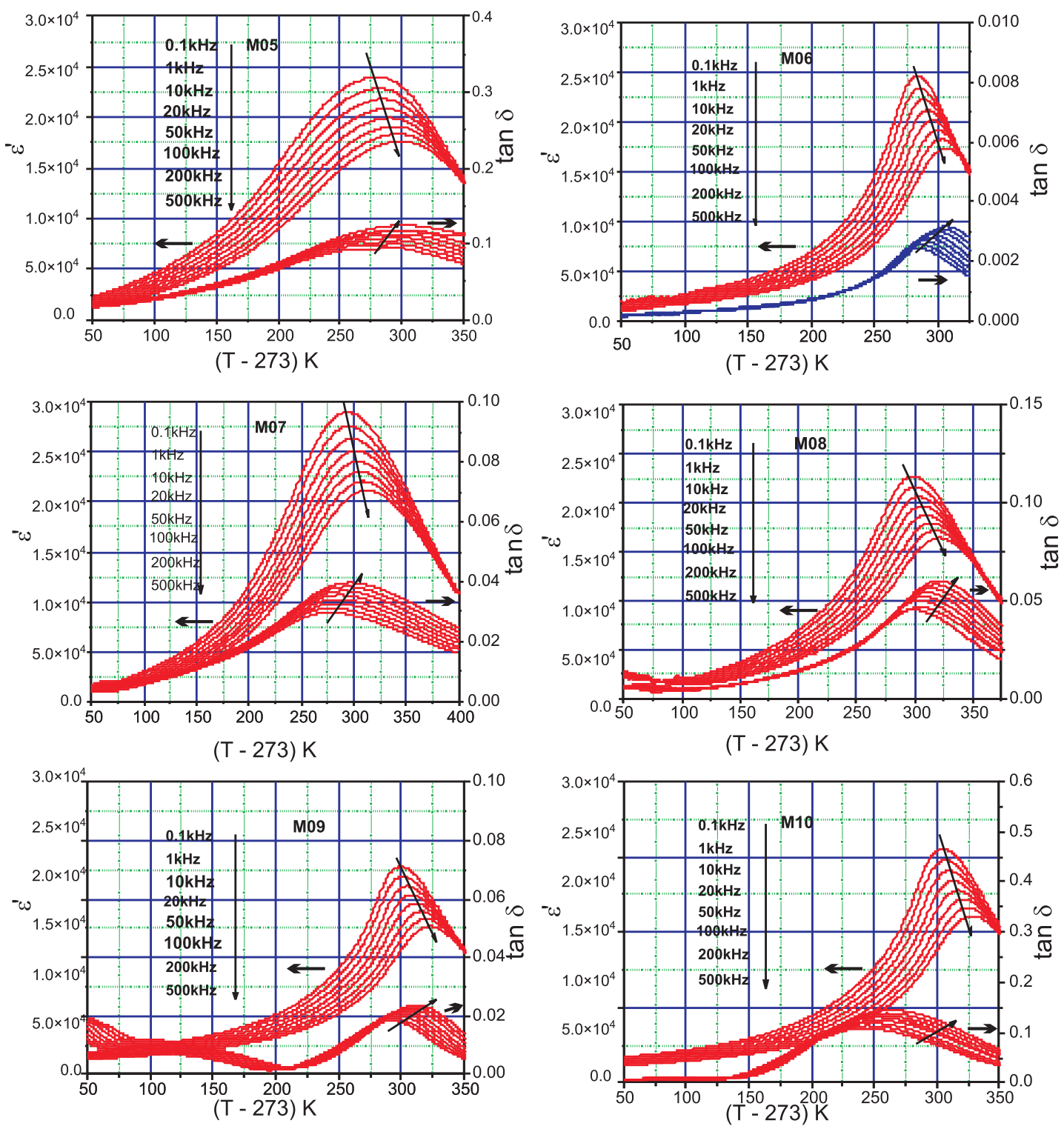

Fig. 1. The temperature dependence of the real part of dielectric permittivity $\varepsilon^{\prime}$ and the dissipation factor tan at various frequency

as in local strain field related to the formation of vacancies in the crystalline structure of materials and/or with the different valences and radius of B-site cation [9]. For PZTPMnN-PSbN system, the B-site is occupied by $\mathrm{Mn}^{2+}, \mathrm{Sb}^{3+}, \mathrm{Nb}^{5+}, \mathrm{Zr}^{4+}$ and $\mathrm{Ti}^{4+}$. Both of $\mathrm{Mn}^{2+}$ and $\mathrm{Sb}^{3+}$ have the ionic radii rather similar: $\mathrm{Mn}^{2+}(0.08 \mathrm{~nm}), \mathrm{Sb}^{3+}(0.082 \mathrm{~nm})$, as substituted on $\mathrm{Nb}^{5+}(0.069 \mathrm{~nm}), \mathrm{Zr}^{4+}(0.079 \mathrm{~nm})$ or $\mathrm{Ti}^{4+}(0.068 \mathrm{~nm})[10]$. Thus, the degree 
of disorder in this system is mainly caused by the difference of valences of $\mathrm{Mn}^{2+}$ and $\mathrm{Sb}^{3+}$ with $\mathrm{Zr}^{4+} / \mathrm{Ti}^{4+}$.

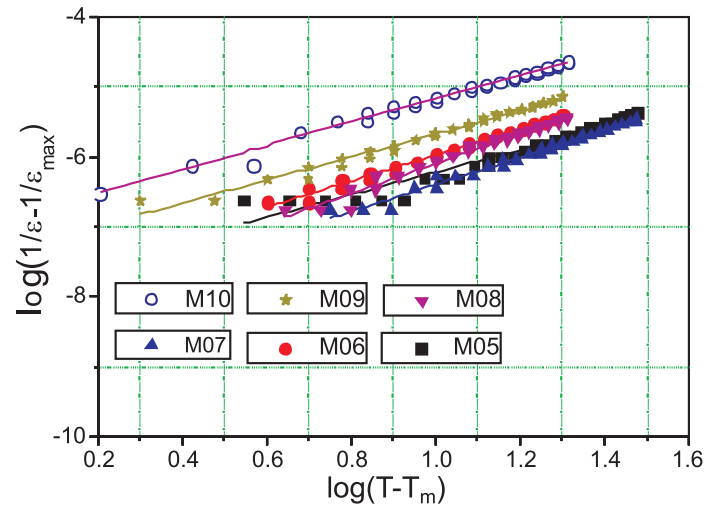

Fig. 2. Dependence of $\log \left(1 / \varepsilon-1 / \varepsilon_{\max }\right)$ on $\log \left(\mathrm{T}-T_{m}\right)$ for PZT-PMnN-PSbN ceramics at frequency of $1 \mathrm{kHz}$

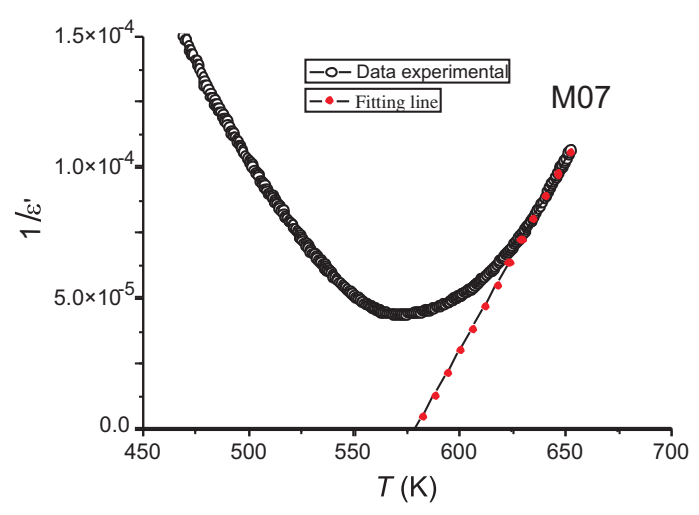

Fig. 3. Curie-Weiss dependence of the permittivity of the M07 sample at temperature much higher than $T_{m}$.

Fig. 3 presents a Curie-Weiss dependence $1 / \varepsilon$ ' of the M07 sample. It is clearly seen that at the temperature region far above $T_{m}$ the dependence fitted well to a linear line. It is supposed to be related with an appearance of the paraelectric phase in the sample. The linear line has cut the $1 / \varepsilon(\mathrm{T})$ curve at a point called as Burns temperature $T_{B}$, the temperature at which the disorder nanoclusters start to appear with cooling down the sample. The values $T_{B}$ given from fitting are also presented in Table 1 . The obtained results suggested that in the diffuse phase transition materials the ferroelectric disorder nanoclusters could exist in a temperature region much higher than the $T_{c}$ evaluated from Curie-Weiss relationship.

\section{III.2. Cole-Cole diagrams}

Fig. 4 show the frequency dependence of real and imaginary parts of dielectric permitivity at different temperatures, of M07 sample for example. From this, the $T_{m}$ at different frequencies can be determined. Table 2 listed the temperatures $T_{m}$ estimated at different frequencies, for PZT-PMnN-PSbN relaxor ferroelectrics.

Complex dielectric constant formalism is the most commonly used experimental technique to analyze dynamics of the ionic movement in solids. Contribution of various microscopic elements such as grain, grain boundary and interfaces to total dielectric response in polycrystalline solids can be identified by the reference to an equivalent circuit, which contains a series of array and/or parallel RC element [15]. To study the contribution originated from difference effects, Cole-Cole analyses have been made at difference temperatures.

It was observed that the dielectric constant data at low temperature, i.e., up to about $289^{\circ} \mathrm{C}$, did not take the shape of a semicircle in the Cole-Cole plot and rather showed the straight line with large slope, suggesting the insulating behaviour of the compound at low temperature. It could further be seen that with the increase in temperature, the slope of 
Table 2. Frequency dependence of $T_{m}$ for PZT-PMnN-PSbN relaxor ferroelectrics.

\begin{tabular}{ccccccc}
\hline $\begin{array}{c}\text { Frequency } \\
(\mathrm{kHz})\end{array}$ & \multicolumn{6}{c}{$T_{m}(\mathrm{~K})$} \\
\cline { 2 - 7 } & $\mathrm{M} 05$ & $\mathrm{M} 06$ & $\mathrm{M} 07$ & $\mathrm{M} 08$ & $\mathrm{M} 09$ & M10 \\
\hline 0.1 & 553 & 555 & 566 & 571 & 573 & 578 \\
1 & 555 & 557 & 569 & 573 & 575 & 580 \\
10 & 558 & 560 & 571 & 575 & 578 & 583 \\
20 & 561 & 563 & 575 & 578 & 581 & 586 \\
50 & 566 & 567 & 577 & 583 & 586 & 588 \\
100 & 568 & 569 & 579 & 585 & 589 & 590 \\
200 & 571 & 571 & 582 & 587 & 591 & 592 \\
500 & 574 & 575 & 585 & 589 & 593 & 595 \\
\hline
\end{tabular}

the lines decreased towards the real $\left(\varepsilon^{\prime}\right)$ axis and at temperature above $289^{\circ} \mathrm{C}$, a semicircle could be traced (Fig. 5).

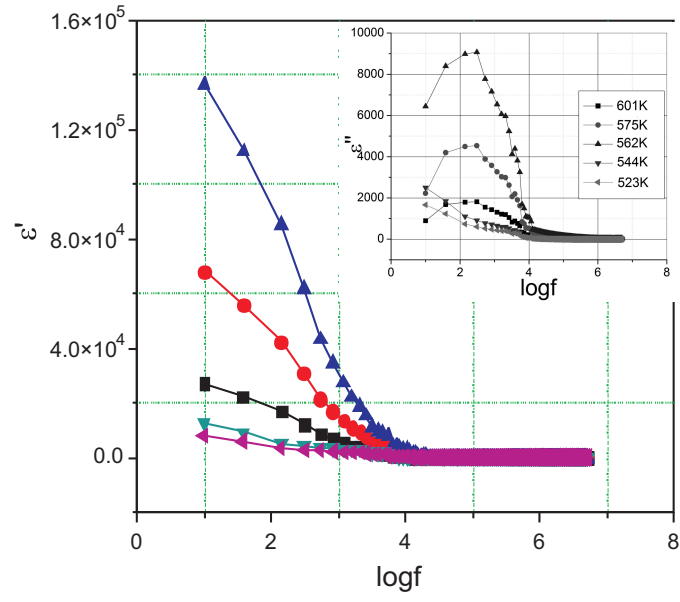

Fig. 4. The frequency dependence of real and imaginary parts of dielectric permitivity of M07 sample at different temperatures

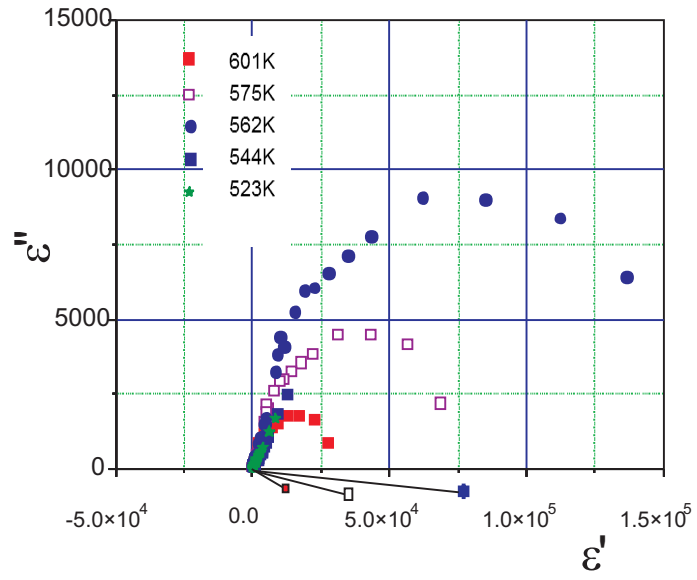

Fig. 5. Cole-Cole diagrams of M07 sample at different temperatures.

The Cole-Cole plot also provides the information about the nature of the dielectric relaxation in the systems. For polydispersive relaxation, which is expected in PZT-PMNPSN, the plots are close to circular arcs with end points on the axis of real and the centre below this axis. The complex dielectric constant in such situations is known to be described by the empirical relation:

$$
\varepsilon *=\varepsilon^{\prime}-i \varepsilon^{\prime \prime}=\varepsilon_{\infty}+\frac{\varepsilon_{s}-\varepsilon_{\infty}}{1+(i \omega \tau)^{1-\alpha}}
$$

where $\varepsilon_{s}$ and $\varepsilon_{\infty}$ are the low- and high-frequency values of $\varepsilon, \alpha$ is a measure of the distribution of relaxation times. The parameter $\alpha$ can be determined from the location of the centre of the Cole-Cole circles, of which only an arc lies above the $\varepsilon^{\prime}$-axis [16]. It is evident 
from the plots that the relaxation process differs from monodispersive Debye process (for which $\alpha=0$ ). The parameter $\alpha$, as determined from the angle subtended by the radius of the circle with the $\varepsilon$ '- axis passing through the origin of the $\varepsilon$ "-axis [17-19], shows a slight increase in the interval $[0.221,0.196,0.183]$ with the decrease of temperature from $601 \mathrm{~K}$ to $562 \mathrm{~K}$, implying a slight increase in the distribution of the relaxation time with decreasing temperature below $T_{m}$. All these analyses suggest again that PZT-PMnN-PSbN is a relaxor ferroelectric.

\section{III.3. The diffuse phase transition (DPT) of PZT-PMnN-PSbN system}

The polar micro regions are suggested to be thermally unstable and flip in all possible directions [14]. Consequently, Debye relation should govern the frequency dependence of the $T_{m}$. For the Debye medium, the relation between $\mathrm{f}$ and $T_{m}$ can be described by the equation

$$
f=f_{0} \exp \left(-\frac{E_{a}}{k_{B} T_{m}}\right)=f_{0} \exp \left(-\frac{T_{0}}{T_{m}}\right)
$$

where, $f$ is the applied frequency, $f_{0}$ is the attempt frequency of a dipole or Debye frequency, $T_{0}=E_{a} / \mathrm{k}_{B}(\mathrm{~K})$ is the equivalent temperature of activation energy $E_{a}$. For a normal ferroelectric, $\mathrm{f}$ is equal to $f_{0}$ at $T_{0}=E_{a} / \mathrm{k}_{B}=0 \mathrm{~K}$; for relaxors, $T_{0}$ may be higher than $0 \mathrm{~K}$.

The experimental results of $T_{m}$ for all the samples at different frequencies are given in Table 2. The experimental data are fitted to the above equation (Fig. 6) and the fitted parameters are given in Table 3.

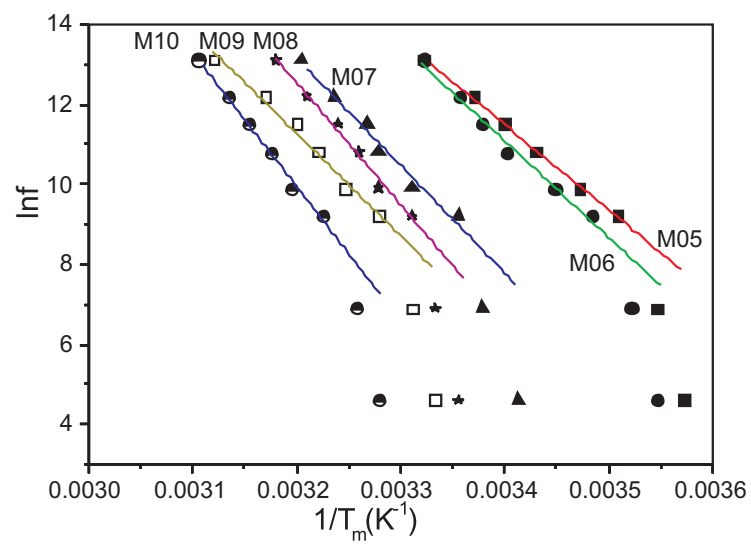

Fig. 6. The fittings to the Debye relation. The solid lines represent the linear fit.

The results of fitting indicate that the dielectric relaxation of PZT-PMnN-PSbN systems did not obey with Debye relation.

To analyze the frequency dependence of $T_{m}$, we can use Vogel-Fulcher law, which is given by:

$$
\phi=\phi_{0} \varepsilon \xi \pi\left(-\frac{\mathrm{E}_{\alpha}}{\kappa_{\mathrm{B}}\left(\mathrm{T}_{\mu}-\mathrm{T}_{\phi}\right)}\right)=\phi_{0} \varepsilon \xi \pi\left(-\frac{\mathrm{T}_{0}}{\left(\mathrm{~T}_{\mu}-\mathrm{T}_{\phi}\right)}\right)
$$


Table 3. Fitting parameters $\left(f_{0}\right.$ and $\left.T_{0}\right)$ to equation (4)

\begin{tabular}{lll}
\hline Sample & $f_{0}(\mathrm{~Hz})$ & $T_{0}(\mathrm{~K})$ \\
\hline M05 & $7.71 \times 10^{50}$ & $3.14 \times 10^{4}$ \\
M06 & $5.92 \times 10^{47}$ & $5.70 \times 10^{4}$ \\
M07 & $1.16 \times 10^{59}$ & $3.85 \times 10^{4}$ \\
M08 & $7.62 \times 10^{67}$ & $4.51 \times 10^{4}$ \\
M09 & $7.83 \times 10^{56}$ & $3.79 \times 10^{4}$ \\
M10 & $8.56 \times 10^{67}$ & $4.65 \times 10^{4}$ \\
\hline
\end{tabular}

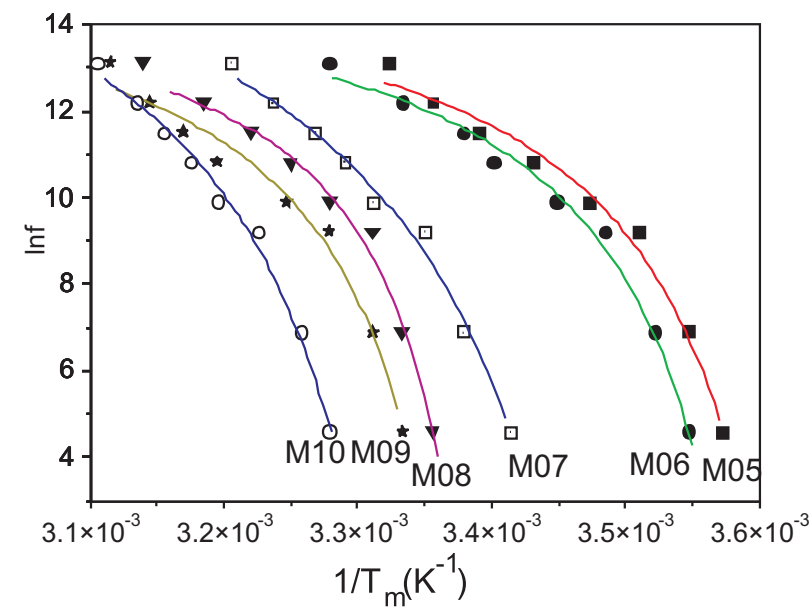

Fig. 7. The frequency dependence of $T_{m}$ fitted against Vogel-Fulcher law.

where $f$ is the frequency for a particular value of $T_{m}, f_{0}$ is the pre-exponential factor, and $T_{f}$ is the freezing temperature. $T_{f}$ is regarded as the temperature where the dynamic reorientation of dipolar cluster polarization can no longer be thermally activated. The fitting curves are shown in Fig. 7 and the fitting parameters $f_{0}, T_{0}$ and $T_{f}$ are listed in Table 4. Even though, $f_{0}$ is in the expected range for all the studied compounds, the $T_{0}$ values (temperature equivalent activation energies) for all the compositions are high, which does not conform with a thermally activated system. This indicates that all the polar clusters in these systems do not freeze at the same temperature rather a range of temperature. An excellent fit of the Vogel-Fulcher law with the experimental data constitutes strong evidence for a static freezing temperature of thermally activated polarization fluctuations in PZT-PMnN-PSbN.

The frequency dependence of $T_{m}$ has also been explained by a super exponential relation called power law as given by

$$
f=f_{0} \exp \left[-\left(\frac{T_{0}}{T_{m}}\right)^{p}\right]
$$

where, $p(>1)$ is associated with the degree of relaxation of the materials. The smaller is the value of $p$, the stronger is the dielectric relaxation of materials. For $p=1$, the equation 
(6) reduced to Debye relation which has the strongest dielectric relaxation and for very high of $p$, the systems behave like normal ferroelectrics where no relaxation phenomena exist.

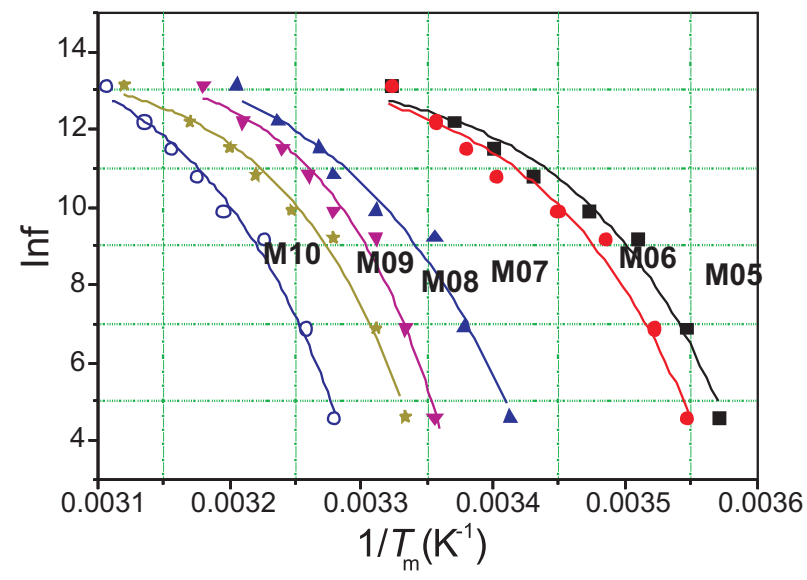

Fig. 8. The frequency dependence of $T_{m}$ to power law.

Fig. 8 show the results fitting of the power law for PZT-PMN-PSN ceramics, following the equation (6).

Excellent fitting of Vogel - Fulcher relation and power relation to experimental data, suggests that the above formalism can be employed to explain the relaxor behavior in PZT-PMN-PSN ceramics which is analogous to that of dipolar glass with polarization fluctuations above a static freezing temperature. The value of phenomenological parameters $T_{0}, f_{0}, T_{f}$ and $\mathrm{p}$ using linear least - squares fit to equations (5) and (6) are consistent with the earlier reports on other similar systems [4-9].

Table 4. The value of fitting parameters to Vogel-Fulcher and power relation

\begin{tabular}{lllllll}
\hline \multirow{2}{*}{ Sample } & $f_{0}(\mathrm{~Hz})$ & \multicolumn{3}{c}{$T_{0}\left({ }^{\circ} \mathrm{C}\right)$} & $T_{f}\left({ }^{\circ} \mathrm{C}\right)$ & $\mathrm{p}$ \\
\cline { 2 - 7 } & $\mathrm{V}-\mathrm{F}$ & power & $\mathrm{V}-\mathrm{F}$ & power & & \\
\hline M05 & $6.6 \times 10^{8}$ & $7.55 \times 10^{8}$ & 561 & 572 & 552 & 33.1 \\
M06 & $3.6 \times 10^{8}$ & $9.42 \times 10^{8}$ & 579 & 575 & 554 & 31.5 \\
M07 & $3.4 \times 10^{8}$ & $1.72 \times 10^{8}$ & 587 & 592 & 565 & 26.8 \\
M08 & $4.6 \times 10^{8}$ & $9.3 \times 10^{8}$ & 583 & 588 & 570 & 40.2 \\
M09 & $7.0 \times 10^{8}$ & $9.3 \times 10^{8}$ & 583 & 592 & 572 & 34.5 \\
M10 & $3.1 \times 10^{8}$ & $18.8 \times 10^{8}$ & 607 & 602 & 577 & 30.7 \\
\hline
\end{tabular}

The value of $f_{0}$ observed in Vogel-Fulcher and power relation agrees well with the values of a classical relaxor, and thereby confirms the existence of interaction between the polar clusters unlike the dipolar glasses. The high values of $\mathrm{p}$ compared to a typical relaxor material suggests that there still exist some normal ferroelectric nature in the samples even though the $\gamma$ values are high enough for a strong relaxor material. 


\section{CONCLUSION}

The rhombohedral polycrystalline PZT-PMnN-PSbN samples were successfully prepared by the Columbite precursor route. All the samples exhibit the relaxor behaviour with diffuse phase transition. Modeling of the dielectric data using modified Curie-Weiss law, Vogel-Fulcher relation and power relation showed a strong evidence for a static freezing temperature of thermally actived polarization fluctuations in the system. Therefore, the dielectric relaxation in the compound is analogous to magnetic relaxation in spin glass system with polarization fluctuations above a static freezing temperature. Complex permittivity analyses indicated that the relaxation should be of non-Debye type.

\section{ACKNOWLEDGMENTS}

This work is supported by the National Foundation for Science and Technology Development (NAFOSTED), No. 103.02.06.09.

\section{REFERENCES}

[1] A. I. Kingon, S. K. Streiffier, C. Basceri and S. R. Summerfelt, Mat. Res. Bull. 21 (1996) 46.

[2] D. L. Polla and L. F. Francis, Mat. Res. Bull. 21 (1996) 59.

[3] S. Saha and T. P. Sinha, J. Phys.; Condens. Matter 14 (2002) 249.

[4] S. K. Sinha, R. N. P. Choudhary, S. N. Choudhary, T. P. Sinha, Materials Letters 51 (2001) 336.

[5] L. E. Cross, Ferroelectrics, 76, 241 (1987); (b) L. E. Cross, Ferroelectrics 151 (1994) 305

[6] S. B. Majumdar, S. Bhattacharyya and R. S. Katiyar, J. Appl. Phys. 99 (2006) 024108.

[7] M. Maglion and M. Belkaoumi, Phys. Rev. B 45 (1992) 2029.

[8] G. Burns and F. H. Dacol, Solid State Commun. 48 (1983) 853.

[9] J.Chen, H.M. Chan, M.P. Harmer, J.Am. Ceram, Soc., 72(5) (1989) 593.

[10] Y. Xu, Ferroelectric, Materials and Their Applications, North Holland (1991)

[11] S.P. Singh, A.K. Singh, D. Pandey, J. Mater. Res. 18 (2003) 2677

[12] S.B. Lee, K.H. Lee, H. Kim, Jpn. J. Appl. Phys. 41 (2002) 5266.

[13] D.R. Chen, Y.Y. Guo, Electron. Element. Mater 1 (1982) 25.

[14] Sinclair D C \& West A R, J Appl Phys, 66 (1989) 38351.

[15] J.R. Macdonald, Impedance Spectroscopy Emphasizing Solid Materials and Systems, Wiley, New York, (1987).

[16] K. S. Cole and R. H. Cole J. Chem. Phys. 9 (1941). 341

[17] G. A. Smolensky, A. I. Agranovskaya, S. N. Popov and V. A. Isupov, Sov. Phys. Tech. Phys. 3 (1958) 1981.

[18] N. Setter and L. E. Cross, J. Appl. Phys. 51 (1980) 4356.

[19] C. A. Randall and A. S. Bhalla, Jpn. J. Appl. Phys. 29 (1990) 327.

[20] X. He, X. Zeng, X. Zheng, P. Qiu, W. Cheng, A. Ding, Journal of Physics: Conference Series 152 (2009) 012068.

[21] K. Bormanis, A. I. Burkhanova, A. V. Shil/nikova, A. Sternberg, S. A. Satarova, Journal of Optoelectronics and Advanced Materials 6(1) (2004) 341.

[22] R. Pirc and R. Blinc, Physical review B76 (2007) 020101.

[23] B. Mihailova, B.J. Maier, N. Waeselmann, C. Paulmann, M. Gospodinov, and U. Bismayer (2011) $12^{\text {th }}$ European Meeting on Ferroelectricity, 3A-2O

Received 01 August 2012. 